\title{
Avaliação de fatores prognósticos da deambulação em crianças com mielomeningocele
}

Luanda André Collange', Roberto S. Martins², Nelci Zanon-Collange Marco T. S. dos Santos², Osmar J. S. Moraes², Renata C. Franco ${ }^{3}$

Serviço de Neurocirurgia do Hospital Santa Marcelina, São Paulo, SP, Brasil

Curso de Fisioterapia do Centro Universitário Nove de Julho (Uninove), São Paulo, SP, Brasil

\section{RESUMO}

Objetivo: Caracterizar o grau de acometimento neurológico, estabelecendo uma correlação entre o nível funcional da lesão e a aquisição da marcha, além de identificar os fatores que interferem nessa capacidade. Método: Foram avaliadas prospectivamente vinte e oito crianças portadoras de mielomeningocele operadas com idade igual ou superior a três anos. Foram determinados o nível funcional da lesão, a aquisição ou não da marcha e os fatores associados por meio das medidas do grau de força muscular nos membros inferiores, presença de deformidades articulares, ocorrência de contratura da musculatura flexora e trato iliotibial, eficácia da reação de equilíbrio e presença de hidrocefalia tratada. Resultados: Todos os fatores avaliados, exceto a hidrocefalia, foram relacionados de forma significativa à aquisição da marcha. Conclusão: A aquisição da marcha em crianças portadoras de mielomeningocele sofre influência de vários fatores, além do nível de acometimento neurológico, o que deve ser considerado no prognóstico e na programação terapêutica.

\section{PALAVRAS-CHAVE}

Mielomeningocele. Distúrbios neurológicos da marcha.

\section{ABSTRACT}

Prognostic factors influencing ambulation in children with myelomeningocele

Objective: To characterize the degree of neurological deficit, establishing a correlation between functional level of the lesion and the gait acquisition and also to identify other factors that interfere with this capacity. Method: Twenty-eight children were evaluated prospectively. The functional level of the lesion was identified and correlated with gait acquisition as well as others associates factors like degree of muscular force, presence of joint deformities, occurrence of contractures, effectiveness of the balance reaction and presence of hydrocephalus. Results: All the evaluated factors, except hydrocephalus, were related with the ability to walk. Conclusion: Several factors, was related to the gait acquisition in children with myelomeningocele, what should be considered in the prognosis and in the therapeutic programming.

\section{KEY WORDS}

Meningomyelocele. Gait disorders, neurologic.

\section{Introdução}

Os defeitos do tubo neural (DFTN) são responsáveis pela maioria das anomalias congênitas do sistema nervoso central (SNC) e resultam de uma falha no desenvolvimento do tubo neural que ocorre entre a terceira e quarta semana de desenvolvimento embrionário ${ }^{14}$. A mielomeningocele (MMC), responsável por cerca de $85 \%$ dos casos de DFTN, é caracterizada pela ausência de fusão dos arcos vertebrais posteriores, com exteriorização de tecido nervoso.

$\mathrm{O}$ quadro clínico das crianças portadoras de MMC tem diversas manifestações com graus variáveis de gravidade. Os pacientes podem apresentar desde um discreto déficit sensitivo-motor de localização distal, incluindo disfunções dos esfíncteres, até uma paralisia

1 Fisioterapeuta do Serviço de Neurocirurgia do Hospital Santa Marcelina, São Paulo, SP.

2 Neurocirurgião do Serviço de Neurocirurgia do Hospital Santa Marcelina, São Paulo, SP.

3 Fisioterapeuta, Professora do Curso de Fisioterapia, Uninove. 
completa abaixo do nível da lesão. A deambulação é uma função importante para essas crianças e sua ausência compromete a independência nas atividades de vida cotidiana, o desenvolvimento escolar e a qualidade de vida $^{12}$. Na maioria das vezes, a deambulação é o objetivo que mais motiva os pais durante o tratamento.

O objetivo deste estudo é avaliar a influência de diversos fatores na aquisição da marcha em 28 crianças portadoras de $\mathrm{MMC}$, por meio da caracterização do nível funcional da lesão e da avaliação do grau de força muscular, das deformidades da coluna, do quadril, do joelho e do tornozelo, da presença de contratura da musculatura flexora do quadril e do trato iliotibial e da presença de hidrocefalia.

\section{Materiais e métodos}

O presente estudo foi aprovado pela Comissão de Ética do Hospital Santa Marcelina, em São Paulo, capital. Os pacientes foram previamente esclarecidos a respeito da metodologia e dos objetivos do estudo e concordaram com a participação por meio do preenchimento de um consentimento informado. No período de 1994 a 2004, foram operadas 115 crianças portadoras de MMC no Hospital Santa Marcelina. Dessa casuística, 28 crianças foram avaliadas de forma prospectiva durante o ano de 2005 e incluídas neste estudo. As crianças foram categorizadas, em relação ao nível de acometimento neurológico da lesão, em níveis torácico, lombar alto, lombar baixo e sacral, segundo a classificação de Hoffer e cols. ${ }^{10}$. O nível de acometimento neurológico foi definido como o nível medular inferior relacionado à inervação de músculos cuja força era suficiente para vencer a gravidade por meio da contração voluntária, quantificada como sendo, no mínimo, grau 3. Com relação à marcha, os pacientes foram classificados em quatro categorias: deambuladores comunitários, deambuladores domiciliares, deambuladores não-funcionais e não-deambuladores ${ }^{16}$. Os seguintes fatores foram avaliados: grau de força muscular nos músculos quadríceps femoral, iliopsoas, tibiais e glúteos; deformidades da coluna, do quadril, do joelho e do tornozelo; presença de contratura da musculatura flexora do quadril; ocorrência de contratura do trato iliotibial; eficácia da reação de equilíbrio e presença de hidrocefalia. A avaliação do grau de força muscular nos músculos dos membros inferiores foi realizada manualmente e graduada de 0 a 5 conforme proposto por Dahl e cols. ${ }^{6}$. As deformidades articulares foram identificadas pela inspeção da postura e avaliação da movimentação passiva das articulações da coluna, quadril, joelhos e tornozelos. Testes específicos foram utilizados para avaliação de contraturas musculares. A presença de contratura da musculatura flexora do quadril foi verificada pelo teste de Thomas e de contratura do trato iliotibial, pelo teste de Ober ${ }^{11}$. $\mathrm{O}$ teste de Thomas é realizado com o paciente em decúbito dorsal horizontal, com os membros inferiores fletidos sobre o quadril. O teste é considerado positivo quando é identificada uma incapacidade em estender a perna sem que haja curvatura da coluna torácica. No teste de Ober, o paciente é posicionado em decúbito lateral com o membro inferior acometido abduzido e apoiado na mão do examinador. $\mathrm{O}$ teste é considerado positivo quando o membro permanece em abdução após o examinador soltá-lo, em decorrência da presença de contratura do trato iliotibial. A eficácia da reação de equilíbrio (controle de tronco) foi testada na postura sentada e considerada positiva quando era identificado um ajuste corporal na tentativa de manter a posição em um assento sem encosto, conforme citado em Bartonek e cols. ${ }^{2}$. A avaliação estatística foi realizada pelo teste de Fischer com um nível de significância de 5\%.

\section{Resultados}

A idade dos pacientes variou de 3 a 11 anos, com média de 6 anos, sendo 11 pacientes do sexo masculino. O gráfico 1 demonstra a distribuição dos pacientes em relação ao nível de acometimento neurológico. O nível funcional torácico foi definido em $32,1 \%(\mathrm{n}=9)$ dos casos; $14,3 \%$ das crianças $(n=4)$ foram classificadas como nível funcional lombar alto; $21,5 \%(n=6)$ como nível funcional lombar baixo e $32,1 \%(\mathrm{n}=9)$ como nível funcional sacral. Nenhuma criança apresentou deambulação domiciliar ou deambulação não-funcional, sendo então classificadas apenas em dois grupos: deambuladores e não-deambuladores.

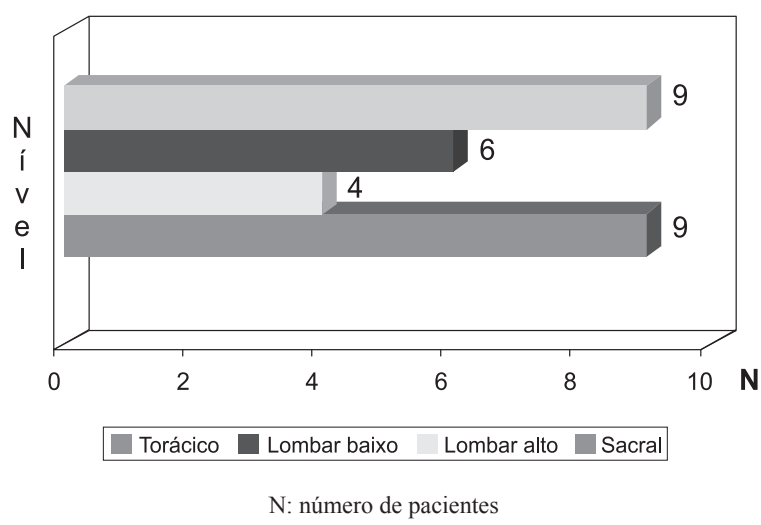

Gráfico 1 - Distribuição dos pacientes de acordo com o nível de acometimento neurológico. 
O gráfico 2 ilustra a distribuição do número de pacientes que apresentaram ou não a deambulação de acordo com o nível de acometimento. Não houve aquisição da marcha nas crianças com nível funcional torácico e lombar alto, enquanto todas as crianças classificadas como portadoras de nível sacral adquiriram a marcha. A aquisição da marcha ocorreu mais freqüentemente nas crianças portadoras de MMC em nível lombar baixo e sacral em comparação com os níveis torácico e lombar alto (teste de Fischer, $\mathrm{p}<0,001$ ).

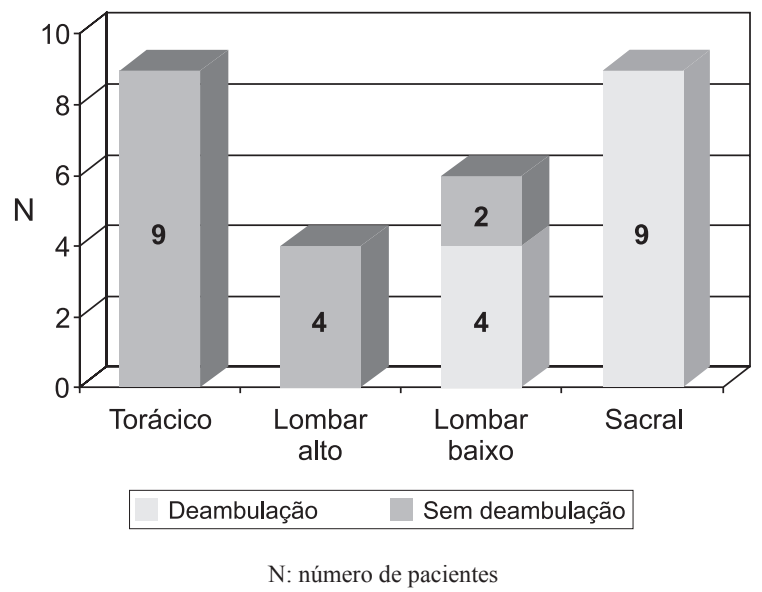

Gráfico 2 - Distribuição dos pacientes com capacidade de deambulação em cada nível de acometimento neurológico.

Os pacientes deambuladores apresentaram grau de força muscular igual ou superior a 4 nos músculos testados, enquanto, entre os não-deambuladores, a maioria $(73,5 \%)$ apresentou grau de força igual ou inferior a 3 nos músculos testados. Foi identificada uma relação significativa entre o grau de força inferior ou igual a 3 nos músculos testados e a ausência de marcha (teste de Fischer, $p<0,0001$ ).

As deformidades da coluna identificadas clinicamente predominaram de forma significativa nos pacientes portadores de lesões de nível mais alto (teste de Fischer, $\mathrm{p}<0,0001)$. Escoliose torácica foi diagnosticada em 6 casos, todos no nível torácico, cifoescoliose em 5 , hipercifose torácica em 1 e hiperlordose lombar em 1 paciente. Todas as crianças portadoras de MMC torácica apresentaram algum tipo de deformidade raquiana. Parte dos pacientes apresentava essa deformidade já ao nascimento. Esse fato ocorreu em 33,3\% dos pacientes com MMC de nível torácico e em $66,7 \%$ das crianças que apresentavam deformidade na coluna e MMC de nível lombar alto.

A única deformidade observada no quadril foi a deformidade em flexão-abdução-rotação externa, presente em nove pacientes, todos não-deambuladores. Uma associação positiva foi identificada entre a presença de deformidade do quadril e a ausência de aquisição da marcha (teste de Fischer, $\mathrm{p}<0,001$ ). A deformidade da articulação do joelho, com postura em flexão dessa articulação, foi observada em 15 pacientes, 7 não-deambuladores. A deformidade de joelho esteve ausente em todos os pacientes deambuladores. A análise estatística mostrou uma relação significativa entre a presença de deformidade em flexão do joelho e a ausência de deambulação (teste de Fischer, $\mathrm{p}<0,01$ ). Todas as crianças não-deambuladoras apresentavam algum tipo de deformidade na articulação do tornozelo.

$\mathrm{O}$ teste de Thomas foi positivo em todas as crianças $(\mathrm{n}=15)$ que não adquiriram a marcha e em uma criança deambuladora. O teste de Ober foi positivo em $94 \%(n=14)$ das crianças não-deambuladoras e negativo em todas as crianças deambuladoras. A positividade desses testes esteve associada de forma significativa com a ausência de aquisição da marcha (teste de Fischer, $\mathrm{p}<0,0001$ ).

A ausência de reação de equilíbrio (controle de tronco) foi identificada em 78,6\% das crianças não-deambuladoras. Todas as crianças deambuladoras apresentaram reação de equilíbrio positiva. A reação de equilíbrio negativa foi relacionada à ausência de deambulação de forma significativa (teste de Fischer, $\mathrm{p}<0,0001$ ).

Dos 15 pacientes que não adquiriram a marcha, 14 (94\%) apresentavam hidrocefalia concomitante previamente tratada com derivação ventriculoperitoneal. A hidrocefalia também foi diagnosticada em $8(61,6 \%)$ dos pacientes deambuladores. Não houve relação entre a presença de hidrocefalia e a aquisição ou não da marcha (teste de Fischer, $\mathrm{p}=0,21$ ). O gráfico 3 demonstra a distribuição das crianças portadoras de hidrocefalia em relação à deambulação.

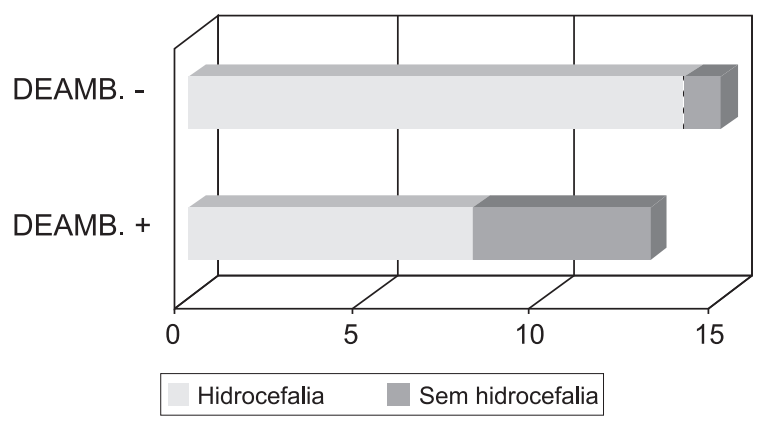

Legenda: Deambulação +: crianças deambuladoras; Deambulação -: crianças não-deambuladoras

Gráfico 3 - Distribuição das crianças com mielomeningocele portadoras de hidrocefalia em relação à deambulação.

\section{Discussão}

Geralmente, o acometimento neurológico das crianças portadoras de MMC é classificado de acordo com a localização em níveis cervical, torácico, lombar alto, lombar baixo e sacral ${ }^{14}$. Apesar de aceito que o nível 
de acometimento esteja relacionado com a função e a aquisição da marcha, resultados obtidos por Bartonek e Saraste $^{3}$ demonstraram que crianças com MMC e paralisia motora similar exibem diferentes funções de deambulação e que outros fatores podem interferir na aquisição da marcha. Conseqüentemente, na avaliação da aquisição da marcha é importante que, além da caracterização do nível de acometimento da lesão, esses fatores descritos sejam também quantificados.

No presente estudo evidenciou-se uma relação direta entre a localização do nível de acometimento neurológico e a capacidade de deambulação, resultado semelhante aos resultados de trabalhos prévios ${ }^{1,2,3,14,15}$. Dos 163 pacientes avaliados por Samuelsson e Skoog ${ }^{15}$, nenhum adquiriu a marcha quando o acometimento neurológico localizava-se no nível torácico. No estudo desses autores, nas lesões de nível lombar, quanto mais inferior o nível funcional, maior o número de pacientes que desenvolveram a marcha, e todos os pacientes com lesões de nível neurológico sacral apresentaram deambulação. De acordo com Vankoski e cols. ${ }^{17}, 80 \%$ das crianças com lesões de nível lombar baixo permanecem como deambuladoras comunitárias durante a vida.

As funções dos músculos quadríceps femoral, iliopsoas, tibiais e glúteos são consideradas determinantes no potencial de deambulação nos pacientes portadores de $\mathrm{MMC}^{1,10,12,16}$. No estudo realizado por McDonald e cols. $^{12}$, o principal fator relacionado à deambulação em 291 crianças com MMC foi o grau de força muscular nesses músculos. Schopler e Menelaus ${ }^{16}$ avaliaram a deambulação em 109 crianças com MMC e observaram que pacientes com graus 4 e 5 de força no músculo quadríceps adquiriram deambulação comunitária. Esses resultados são concordantes com os obtidos no nosso estudo, em que todas as crianças que não adquiriram marcha apresentaram fraqueza da musculatura dos membros inferiores e todas as que a adquiriram apresentaram graus de força 4 e 5 dos músculos dos membros inferiores.

A ocorrência de contraturas de partes moles, principalmente aquelas relacionadas à flexão do quadril e ao trato iliotibial, é um fator importante na influência da aquisição da marcha em crianças com MMC e exige, na maioria das vezes, uma correção cirúrgica ${ }^{8}$. Em nosso estudo verificamos que as contraturas da musculatura flexora do quadril e do trato iliotibial interferem na aquisição da marcha. Bartonek e cols. ${ }^{2}$ observaram, durante 12 anos, 60 pacientes com MMC e verificaram que o desenvolvimento de contratura da musculatura flexora do quadril é um dos fatores responsáveis pela mudança no estado de deambulação desses pacientes. A influência negativa desse tipo de contratura sobre a deambulação dessas crianças também foi descrita por Samuelsson e Skoog ${ }^{15}$.

As deformidades da coluna vertebral em crianças com MMC podem ser congênitas ou adquiridas. A deformidade mais comum é a escoliose, algumas vezes relacionada a alterações do sistema nervoso, como hidromielia e medula ancorada, que causam, nesses casos, uma deformidade progressiva ${ }^{5,14}$. No nosso estudo todos os pacientes que não adquiriram marcha apresentavam deformidades na coluna vertebral. A escoliose tem sido descrita como fator que interfere de forma significativa na aquisição da marcha ${ }^{13,15}$.

No nosso estudo, outras deformidades articulares, especialmente no quadril, joelho e tornozelo, foram relacionadas de forma significativa com a capacidade de deambulação das crianças com MMC. De acordo com Samuelsson e Skoog ${ }^{15}$, a deformidade e a luxação do quadril apresentam influência importante sobre a deambulação nessas crianças. A relação entre a deformidade do joelho em flexão e a capacidade de deambulação é assunto controverso na literatura ${ }^{1,2,15}$. As deformidades na articulação do tornozelo atingem $89 \%$ das crianças com MMC de nível alto ${ }^{4}$. A deformidade do tornozelo é um fator que interfere diretamente na aquisição da marcha, o que pode ser confirmado pelos resultados obtidos em nosso estudo, no qual todos os pacientes que não adquiriram marcha apresentaram esse tipo de alteração.

Aproximadamente $20 \%$ a $30 \%$ das crianças portadoras de MMC desenvolvem sintomas acentuados, relacionados às disfunções de tronco, e alterações importantes, relacionadas ao controle postural e ao equilíbrio, levando a um retardo nas aquisições motoras e à limitação da mobilidade ${ }^{6,14}$. A influência da reação de equilíbrio, como a identificada em nosso estudo, foi também relatada por Bartonek e Saraste ${ }^{2}$ e Gupta e cols. ${ }^{9}$.

A presença de hidrocefalia tem sido relacionada de forma significativa com deambulação limitada em crianças portadoras de $\mathrm{MMC}^{14}$. Além da lesão de constituintes das vias motoras, em alguns casos a hidrocefalia pode influenciar na aquisição da marcha de forma secundária pelo comprometimento da cognição ${ }^{7}$. Em nosso estudo, a hidrocefalia não influenciou a aquisição da marcha, possivelmente em conseqüência da realização precoce da derivação liquórica dessas crianças. Segundo Fobe e cols. ${ }^{7}$, em crianças com MMC e hidrocefalia, existe uma correlação direta entre o nível de acometimento motor medular e os resultados de testes cognitivos; e quanto mais precoce o tratamento da hidrocefalia nesses casos, melhores são os resultados obtidos. 


\section{Conclusão}

Em crianças com MMC, o nível de acometimento neurológico afeta a condição de deambulação. Outras variáveis significativas para o estabelecimento de um prognóstico com relação à deambulação é o grau de força muscular nos músculos dos membros inferiores, as deformidades da coluna, do quadril, do joelho e do tornozelo, a presença de contratura da musculatura flexora do quadril, a ocorrência de contratura do trato iliotibial e eficácia da reação de equilíbrio. Nos casos avaliados, não houve influência da hidrocefalia sobre a aquisição da marcha.

\section{Referências}

1. ASHER M, OLSON J: Factors affecting the ambulatory status of patients with spina bifida cystica. J Bone Joint Surg (Am) 65:350-6, 1983.

2. BARTONEKA, SARASTE H, SAMUELSSON L, SKOOG M: Ambulation in patients with myelomeningocele: a 12- year follow-up. J Ped Orthop 19:2002-6, 1999.

3. BARTONEKA, SARASTE H: Factors influencing ambulation in myelomeningocele: a cross-sectional study. Dev Med Child Neurol 43:253-60, 2001.

4. BROUGHTON NS, GRAHAN G, MENELAUS MB: The high incidence of foot deformity in patients with high-level spina bifida. J Bone Joint Surg (Am) 76:548-50, 1994.

5. CALDARELLI M, DI ROCCO C, LA MARCA F: Treatment of hydromyelia in spina bifida. Surg Neurol 50:411-20, 1998.

6. DAHL M, AHLSTEN G, CARLSON H, RONNE-ENGSTRÖM E, LAGERKVIST B, MAGNUSSON G: Neurological dysfunction above cele level in children with spina bifida cystica: a prospective study to three years. Dev Med Child Neurol 37:30-40, 1995

7. FOBE J, RIZZO APP, SILVA IM, Da SILVA SPM, TEXEIRA CE, SOUZA AMC, FERNANDES A: QI em pacientes com hidrocefalia e mielomeningocele. Arq Neuropsiquiatr (São Paulo) 57:44-50, 1999.
8. GABRIELI APT, VANKOSKI S, DIAS LS, MILANI C, LOURENÇO A, FILHO JL: Análise laboratorial de marcha na mielomeningocele de nível lombar baixo e instabilidade unilateral do quadril. Acta Ortop Bras 12:91-8, 2004.

9. GUPTA RT, VANKOSKI S, NOVAK RA, DIAS LS: Trunk kinematics and the influence on valgus knee stress in persons with high sacral level myelomeningocele. J Pediatr Orthop 25:89-94, 2005

10. HOFFER MM, FEIWELL E, PERRY R, PERRY J, BONNETT $C$ : Functional ambulation in patients with myelomeningocele. J Bone Joint Surg (Am) 55:137-48, 1973.

11. HOPPENFELD S: Propedêutica Ortopédica. São Paulo: Atheneu, 1999. pp. 162-6.

12. McDONALD CM, JAFFE KM, MOSCA VS, SHURTLEFF DB: Ambulatory outcome of children with myelomeningocele: effect of lower-extremity muscle strength. Dev Med Child Neurol 33:482-90, 1991

13. MÜLLER BE, NORDWALL A, VON WENDT L: Influence of surgical treatment of scoliosis in children with spina bifida on ambulation and motoric skills. Acta Paediatr 81:173-6, 1992.

14. NORRLIN S, STRINNHOLM M, CARLSSONM, DAHL M: Factors of significance for mobility in children with myelomeningocele. Acta Paediatr 92:204-10, 2003.

15. SAMUELSSON L, SKOOG M: Ambulation in patients with myelomeningocele: a multivariate statistical analysis. J Ped Orthop 8:569-75, 1988

16. SCHOPLER SA, MENELAUS M: Significance of the strength of the quadriceps muscles in children with myelomeningocele. J Pediatr Orthop 7:507-12, 1987.

17. VANKOSKI SJ, SARWARK JF, MOORE C, DIAS L: Characteristic pelvic, hip, and knee kinematic patterns in children with lumbosacral myelomeningocele. Gait Posture 3:51-7, 1995.

Original recebido em abril de 2006

Aceito para publicação em agosto de 2006

\section{Endereço para correspondência}

Luanda André Collange

Av. Eulina, 217

02755-140 - São Paulo, SP

E-mail:luandacollange@ig.com.br 